$$
\begin{gathered}
\text { NAG3-548 } \\
\angle \text { EW/S GRAS } \\
\text { A-34-CR } \\
19669 \\
035
\end{gathered}
$$

\title{
NAVIER-STOKES ANALYSIS OF TURBOMACHINERY BLADE EXTERNAL HEAT TRANSFER
}

\author{
Rama S. R. Gorla \\ Professor of Mechanical Engineering \\ Cleveland State University \\ Cleveland, Ohio 44115
}




\title{
Navier-Stokes Analysis of Turbomachinery Blade External Heat Transfer
}

\begin{abstract}
The two-dimensional, compressible, thin-layer Navier-Stokes and energy equations were solved numerically to obtain heat transfer rates on turbomachinary blades. The Baldwin-Lomax algebraic model and the $q-\omega$ Low Reynolds Number Two-Equation model were used for modeling of turbulence. For the numerical solution of the governing equations a four-stage Runge-Kutta solver was employed. The turbulence model equations were solved using an implicit scheme. Numerical solutions are presented for two-dimensional flow within two vane cascades. The heat transfer results and the pressure distributions were compared with published experimental data. The agreement between the numerical calculations and the experimental values were found to be generally favorable. The position of transition from laminar to turbulent flow was also predicted accurately.
\end{abstract}




\section{Nomenclature}

$A R C \quad$ Surface arc length

a Sonic speed

C Axial chord

$c_{1} \quad$ Constant in the $q-\omega$ equations $=0.405 \mathrm{D}+0.045$

$c_{2} \quad$ Constant in the $q-\omega$ equations $=0.92$

$c_{\mu} \quad$ Constant in the $q-\omega$ equations $=0.9$

$D \quad$ Damping factor in the $q-\omega$ equations

$e \quad$ Total energy per unit volume

$H \quad$ Heat transfer coefficient

$H_{0}$. Reference heat transfer coefficient

$J \quad$ Jacobian of transformation

$k \quad$ Turbulent kinetic energy

$M \quad$ Mach number

$p \quad$ Pressure

$\mathcal{P} \quad$ Production of turbulence

$\operatorname{Pr} \quad$ Prandtl number

$q \quad$ Variable in the $q-\omega$ equations $=\sqrt{k}$

$\mathrm{Q} \quad$ Vector of dependent variables

Re Reynolds number

$S \quad$ Surface distance

St Stanton number

$t \quad$ Time

$T \quad$ Temperature

Tu Turbulence intensity

$U, V$ Contravariant components of the velocity

$u, v \quad$ Cartesian components of the velocity 


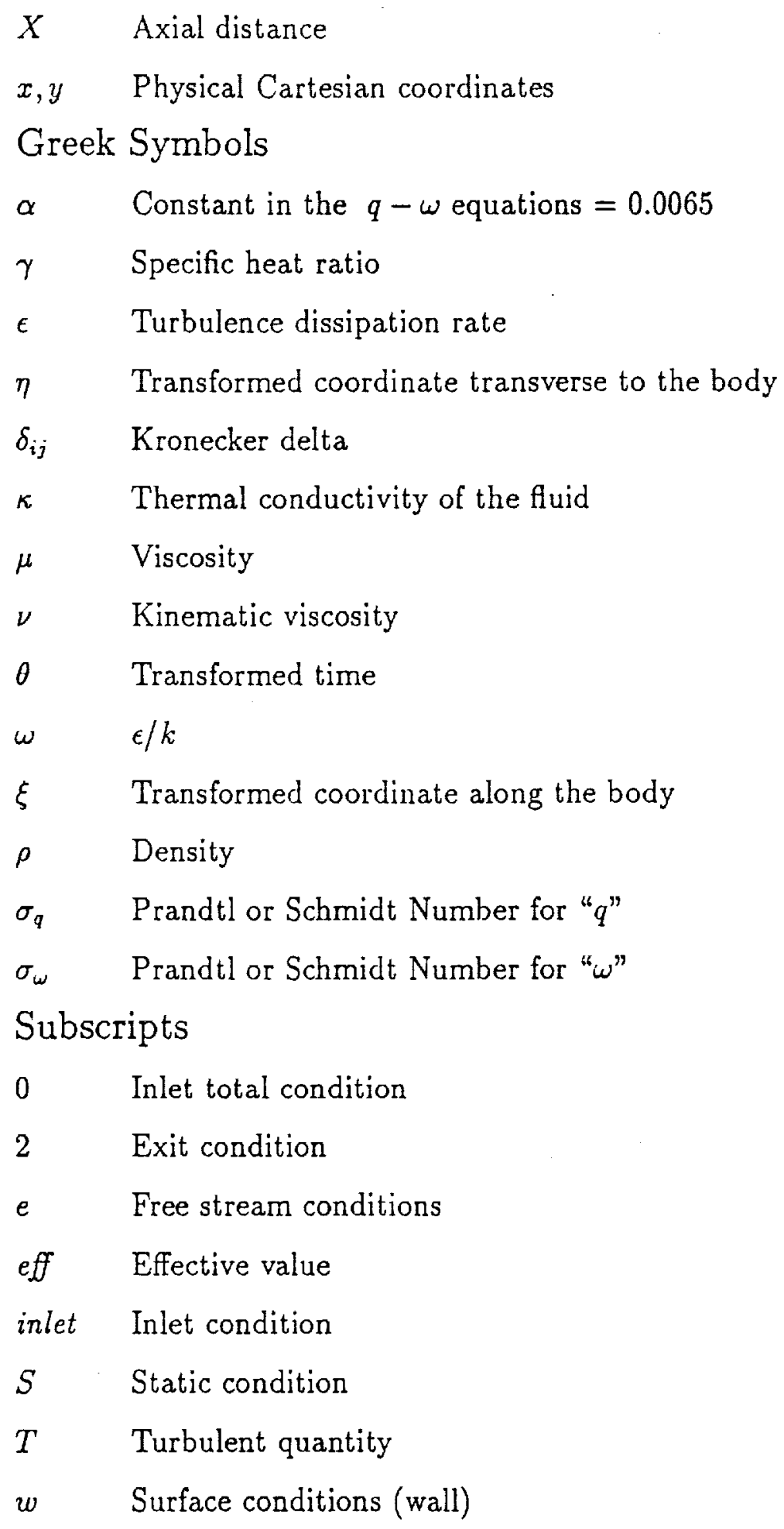




\section{Introduction}

Modern gas turbines operate at high temperature and pressure to achieve improved efficiencies. The design of such engines for extended periods of operation requires an extensive knowledge of heat transfer rates. Integral boundary layer methods represent the simplest methods which may be used to determine heat transfer but, because of the many assumptions made in their development, have a limited range of applications. Therefore, these methods are often employed in preliminary design applications. Differential boundary layer methods using parabolic solvers such as GENMIX [1] are more of a standard practice for calculation of heat transfer. A popular computer code for this purpose, namely STAN5 [2], was developed over many years along with a parallel experimental program on the fundamentals of convective heat transfer. This code incorporates many empirically obtained tools to make the modeling of such phenomena as laminar/turbulent transition and the effect of turbulence possible. The differential-method has a wider range of application compared to the integral method. The method, however, fails near stagnation and separation points. It also requires the specification of starting profiles near the leading edge and the prescription of the flow velocity or the pressure distribution at the edge of the boundary layer. This boundary specification needs to be performed for the turbulence model parameters if one opts to use a model which employs differential equations (e.g. two- equation models, Reynolds stress models, etc.). Many suggestions have been made regarding the form and the manner in which such boundary conditions are to be specified [3-7]. In view of the fact that the solution is sensitive to the starting profile and to the free stream boundary condition, an alternative method is desirable.

In a blade cascade, the flow conditions are very complex and many complicating phenomena are at work simultaneously. A list of the more important flow phenom- 
ena and blade characteristics affecting the blade heat transfer would contain: laminar, transitional and turbulent flows; stagnation flow; acceleration and deceleration; free stream turbulence; separation; curvature; surface roughness; Mach number effects; transpiration; shock boundary layer interaction; and flow unsteadiness. It is desirable that the method of analysis have the capability to account for the above effects. The ideal approach would be to solve the unsteady Navier-Stokes equations. However, the large computer time and memory requirements make this approach as yet impossible. The blade-to-blade solution of the Reynolds averaged Navier-Stokes equations along with a suitable model of turbulence is an alternative that can be utilized as a design tool. Some authors have calculated friction and/or heat transfer using time-marching methods originally devised for inviscid Euler calculations. Carter et al. [8] and Marconi and Wilson [9] have discussed the contamination of the velocity and temperature profiles in the boundary layer which comes about as a result of the smoothing operation performed to prevent oscillations and odd-even decoupling. In references [8-10] special care was taken to minimize this problem.

In the work, presented herein, the two-dimensional, compressible, Navier-Stokes and energy equations were solved using a time-marching scheme to obtain heat transfer rates on turbomachinery blades. For the turbulent closure problem, two models were employed, namely, Baldwin-Lomax algebraic model and $q-\omega$ low Reynolds number two-equation model. The formulation of the problem and the turbulence models employed, are discussed next, numerical issues and results are presented subsequently.

\section{Formulation}

As discussed in the introduction, direct solution of the Navier-Stokes equations is impractical because of the very small grid size requirement. To circumvent this 
problem, the mass-averaged (Favre) Navier-Stokes equations are used. The massweighted averaging helps to simplify the formulation of the turbulent compressible flow but it does not eliminate the need for closure assumptions. In this work, the Baldwin-Lomax algebraic model [11] and the $q-\omega$ low Reynolds Number model of Coakley [12] were employed.

\section{Governing Equations}

The mass-weighted time-averaged governing equations in the conservation law form in body-fitted coordinates as given in [13] will be given. The general transformation is:

$$
\theta=t ; \quad \xi=\xi(x, y) ; \quad \eta=\eta(x, y)
$$

which is used to transform the physical domain $(x, y)$ to the computational domain $(\xi, \eta)$. The governing equations in the transformed coordinates are:

$$
\frac{\partial \mathbf{Q}}{\partial \theta}+\frac{\partial \mathbf{E}}{\partial \xi}+\frac{\partial \mathbf{F}}{\partial \eta}=\left(\frac{\partial \mathbf{R}}{\partial \xi}+\frac{\partial \mathrm{S}}{\partial \eta}\right)
$$

where

$$
\begin{array}{r}
\mathrm{Q}=J^{-1}\left(\begin{array}{c}
\rho \\
\rho u \\
\rho v \\
e
\end{array}\right) ; \mathrm{E}=J^{-1}\left(\begin{array}{c}
\rho U \\
\rho u U+\xi_{x} p \\
\rho v U+\xi_{y} p \\
U(e+p)
\end{array}\right) ; \mathrm{F}=J^{-1}\left(\begin{array}{c}
\rho V \\
\rho u V+\eta_{x} p \\
\rho v V+\eta_{y} p \\
V(e+p)
\end{array}\right) \\
\mathrm{R}=J^{-1}\left(\begin{array}{c}
0 \\
\xi_{x} \tau_{x x}+\xi_{y} \tau_{x y} \\
\xi_{x} \tau_{x y}+\xi_{y} \tau_{y y} \\
\xi_{x} R_{4}+\xi_{y} S_{4}
\end{array}\right) ; \mathrm{S}=J^{-1}\left(\begin{array}{c}
0 \\
\eta_{x} \tau_{x x}+\eta_{y} \tau_{x y} \\
\eta_{x} \tau_{x y}+\eta_{y} \tau_{y y} \\
\eta_{x} R_{4}+\eta_{y} S_{4}
\end{array}\right)
\end{array}
$$

The transformed viscous terms are:

$$
\tau_{x x}=\mu_{e f f}\left[\frac{4}{3}\left(\xi_{x} u_{\xi}+\eta_{x} u_{\eta}\right)-\frac{2}{3}\left(\xi_{y} v_{\eta}\right)\right]-\frac{2}{3} \rho k
$$




$$
\begin{gathered}
\left.\tau_{x y}=\mu_{e f f}\left[\left(\xi_{y} u_{\xi}+\eta_{y} u_{\eta}\right)+\xi_{x} v_{\xi}+\eta_{x} v_{\eta}\right)\right] \\
\tau_{y y}=\mu_{e f f}\left[\frac{4}{3}\left(\xi_{y} u_{\xi}+\eta_{y} v_{\eta}\right)-\frac{2}{3}\left(\xi_{x} u_{\eta}\right)\right]-\frac{2}{3} \rho k \\
R_{4}=u \tau_{x x}+v \tau_{y y}+\left(\frac{\mu}{P r}+\frac{\mu_{T}}{P r_{T}}\right)(\gamma-1)^{-1}\left(\xi_{x} \partial_{\xi} a^{2}+\eta_{x} \partial_{\eta} a^{2}\right) \\
S_{4}=u \tau_{x y}+v \tau_{x y}+\left(\frac{\mu}{P r}+\frac{\mu_{T}}{P r_{T}}\right)\left(\xi_{y} \partial_{\xi} a^{2}+\eta_{y} \partial_{\eta} a^{2}\right)
\end{gathered}
$$

Constant values of $\operatorname{Pr}=0.71$ and $\operatorname{Pr}_{T}=0.9$ were used for all the calculations. The temperature differences within the cascades are not large enough to warrant the use of a variable Prandtl number. Comparing the results obtained using both a variable and a constant value of $\operatorname{Pr}_{T}$, Boyle [10] concluded that the differences in the results were small. This is understandable if one realizes that the contribution of the term $\mu_{T} / P r_{T}$, in the region very close to the wall is very small. This also happens to be the region in which variable Prandtl number models show a large value for $\operatorname{Pr}_{T}$.

The thin-layer approximation, as discussed by Baldwin and Lomax [11], was used to simplify the coding.

\section{Body-Fitted Grid Generation}

The quality of the computational grid is vital to the success of the numerical computations. The grid has to be fine enough in locations where large gradients are present (such as near the walls and in the vicinity of the stagnation points). They also need to be coarse where these gradients are not as severe (free stream, far wake, far upstream) to make the computations more economical.

There are a number of different methods for generating such grids. Algebraic, hyperbolic and elliptic methods are common ways of accomplishing the task. In this work elliptic mesh generation is employed to construct a C-type grid. The advantage of this method is that the grid so generated is smooth and free of discontinuities. A code called GRAPE which was developed by R. Sorenson [14] is employed for this 
purpose.

\section{Baldwin-Lomax Zero-Equation Turbulence Model}

This model [11] is a two-layer model that divides the boundary layer into an inner layer where the viscous effects are dominant and an outer layer where the turbulence effects are of more significance. This model is based on the well-documented CebeciSmith [15] model. The inner region is modeled in nearly the same way as the CebeciSmith model. It uses the magnitude of the vorticity in the inner region in contrast to the Cebeci-Smith model which uses the shearing strain. The need to calculate the boundary layer and the displacement thickness is eliminated by replacing the length scale by one which is easily computed. The free stream turbulence is not a variable in this model, therefore the influence of the free stream turbulence on the heat transfer rate cannot be modeled using this model. Recently Boyle [10] has made modifications to this model to account for several effects not included in the original model. This model can be easily programmed and is popular in the CFD community.

\section{q- $\omega$ Low Reynolds Number Turbulence Model}

It is well known that the heat transfer on turbomachinery blades is affected by the level of the free stream turbulence. The $q-\omega$ model of Coakley [12] was chosen to help simulate the effects of free stream turbulence and model transition. The constants which determine the high Reynolds number behavior of the model were determined as a result of the direct transformation of the high Reynolds number model proposed by Jones and Launder from $k$ and $\epsilon$ to $q=\sqrt{k}$ and $\omega=\epsilon / k$ 
with the kinetic energy assumed constant in the diffusion terms. The low Reynolds number part of the model was constructed by the choice of damping functions that reasonably produced the skin friction and profiles of velocity and kinetic energy in calculation of low-speed boundary layer and pipe flows. As described by Coakley, the unusual choice of $q$ in place of $k$ eliminates the need for additional terms to balance molecular diffusion. The chosen turbulence model has superior numerical behavior compared to the $k-\epsilon$ model. This can be understood if one considers the behavior of those parameters close to a wall. Close to a wall $k$ and $\epsilon$ are both proportional to $y^{2}$, but $q$ is proportional to $y$ and $\omega$ is constant making the present model less stiff.

The model equations cast in the conservation form are:

$$
\frac{\partial \mathrm{Q}}{\partial \theta}+\frac{\partial \mathrm{E}}{\partial \xi}+\frac{\partial \mathrm{F}}{\partial \eta}=\left(\frac{\partial \mathrm{R}}{\partial \xi}+\frac{\partial \mathrm{T}}{\partial \eta}\right)+\mathrm{S}
$$

where the flux vectors are defined as

$$
\begin{gathered}
\mathrm{Q}=J^{-1}\left(\begin{array}{c}
\rho q \\
\rho \omega
\end{array}\right) ; \mathrm{E}=J^{-1}\left(\begin{array}{c}
\rho U q \\
\rho U \omega
\end{array}\right) \\
\mathbf{F}=J^{-1}\left(\begin{array}{c}
\rho V q \\
\rho V \omega
\end{array}\right) ; \quad \mathbf{R}=J^{-1}\left(\begin{array}{c}
\left(\mu+\frac{\mu \tau}{\sigma_{q}}\right)\left(q_{\xi} m_{1}+q_{\eta} m_{2}\right) \\
\left(\mu+\frac{\mu r}{\sigma_{\omega}}\right)\left(\omega_{\xi} m_{1}+\omega_{\eta} m_{2}\right)
\end{array}\right) \\
\mathrm{T}=J^{-1}\left(\begin{array}{c}
\left(\mu+\frac{\mu r}{\sigma_{q}}\right)\left(q_{\xi} m_{2}+q_{\eta} m_{3}\right) \\
\left(\mu+\frac{\mu r}{\sigma_{\omega}}\right)\left(\omega_{\xi} m_{2}+\omega_{\eta} m_{3}\right)
\end{array}\right)
\end{gathered}
$$

In the above, $m_{1}=\xi_{x}{ }^{2}+\xi_{y}{ }^{2}, m_{2}=\xi_{x} \eta_{x}+\xi_{y} \eta_{y}, m_{3}=\eta_{x}{ }^{2}+\eta_{y}{ }^{2}$.

The source terms of the model are:

$$
\mathrm{S}=J^{-1}\left(\begin{array}{l}
1 / 2\left(C_{\mu} D \mathcal{P} / \omega^{2}-1-\frac{2}{3} \mathcal{D} / \omega\right) \rho \omega q \\
\left(C_{1}\left(C_{\mu} \mathcal{P} / \omega^{2}-\frac{2}{3} \mathcal{D} / \omega\right)-C_{2}\right) \rho \omega^{2}
\end{array}\right)
$$

in which

$$
D=\left[1-\exp \left(-\alpha R e_{T}\right)\right]
$$


where the term $\operatorname{Re}_{T}=q y / \nu$ is the turbulence Reynolds number. In tensor notation,

$$
\mathcal{D}=u_{k, k}
$$

is the divergence of the velocity field,

$$
\mathcal{S}=\left(u_{i, j}+u_{j, i}-\frac{2}{3} \delta_{i, j} u_{k, k}\right) u_{i, j}
$$

is the strain rate invariant, and

$$
\mathcal{P}=\mu_{T} \mathcal{S}-\frac{2}{3} \rho k \mathcal{D}
$$

is the rate of production of turbulent kinetic energy.

The turbulent viscosity is defined as:

$$
\mu_{T}=C_{\mu} D \frac{\rho k}{\omega}
$$

The process of transition, as simulated by the low Reynolds number two-equation models is described by Patankar and Schmidt [6]. Their description of the process is as follows: The convection and diffusion of kinetic energy into the boundary layer increases the "production" term in the modeled kinetic energy equation which in turn causes a rapid increase in $k$ or $q$ and $\mu_{T}$. This is how the transition process is simulated. The process then slowly decays and stabilizes due to the low Reynolds number functions and the influence of the boundary conditions. This they conclude, limits the usefulness of such models to flows for which the free stream turbulence is the cause of transition.

The method of solution adopted was an implicit scheme and is discussed in a later section.

\section{Boundary Conditions}


Chima [13] presents the details on the stable method of specification/calculation of the boundary conditions for the solution of the mean-flow equations.

The boundary conditions on $q$ and $\omega$ were set up in the following manner:

Inlet- At the inlet of the calculation domain the value of $q$ is specified using the experimental conditions, namely:

$$
q=\sqrt{k}=\sqrt{1.5\left(T u_{\text {inlet }} U_{\text {inlet }}\right)^{2}}
$$

The value of $\omega$ is specified in the following manner: First a value for $\epsilon$ is estimated. To accomplish this, an estimate for the dissipation length scale is required. This length scale is usually not reported as a part of the experimental conditions. For cascade conditions the dissipation length scale is assumed to be equal to a certain percent of the pitch. For example Hah [16] assumed a dissipation length scale equivalent to $1.0 \%$ of the pitch. This assumption was made in the course of the calculations performed in this work. With the assumed length scale, one now proceeds to make an estimate of the rate of dissipation at the inlet.

$$
\epsilon=k^{3 / 2} / \ell \text { and } \omega=\epsilon / k
$$

Wall- The boundary conditions for the turbulence parameters on the wall are:

$$
q=0 \text { and } \frac{\partial \omega}{\partial n}=0
$$

where $n$ is in the direction normal to the wall.

Exit- The exit boundary conditions were computed by assuming the second derivative of $q$ and $\omega$ to be zero in the streamwise direction.

Periodic Boundary- The periodic boundary was treated as an interior point. It should be noted that the boundary conditions were imposed explicitly.

\section{Numerical Scheme}


The quasi-three-dimensional viscous code (RVCQ3D) developed by Chima [13] is utilized to predict the mean flow. The code employs an explicit, finite-difference, multi-stage, Runge-Kutta algorithm. The multi-stage Runge-Kutta scheme developed by Jameson, Schmidt, and Turkel [17] is used to advance the flow equations in time from an initial guess to a steady state. Local time stepping and residual smoothing are used to help stabilize the scheme and accelerate convergence. The convective terms are evaluated at every stage of the scheme. The diffusive and dissipative terms are evaluated at the first and second stage of the scheme to improve stability and convergence. The residual smoothing is also performed at all of the four stages of the Runge-Kutta scheme. The Beam-Warming approximate factorization [18] implicit scheme is used for the solution of the $q-\omega$ model equations. This results in a two-by-two system of coupled equations. The equations are coupled through the Jacobian of the source term matrix. This matrix was replaced by a simple diagonal form suggested by Coakely [12], namely:

$$
\mathrm{D}=\lambda \mathbf{I}
$$

where

$$
\lambda=2 \nu^{\prime} /\left(\xi_{x}^{2}+\xi_{y}^{2}\right)
$$

and

$$
\nu^{\prime}=\frac{J}{\rho}\left(\gamma\left(\frac{\mu}{P r}+\frac{\mu_{T}}{P r_{T}}\right)\right)
$$

Coakley reports that the above choice maintains the diagonal dominance of the implicit tridiagonal solver.

This resulted in decoupling of the model equations and thereby a reduction of the computing time per iteration.

\section{Artificial Dissipation}


As discussed previously, when using central differencing in the convection terms it is necessary to add artificial damping to prevent the occurrence of instability in the solution algorithm.

It was noticed in the course of this work and it has been reported by others including Marconi and Wilson [9] and Davis, $\mathrm{Ni}$ and Carter [8], that the artificial dissipation has a detrimental effect on the prediction of heat transfer and wall shear stress. It has to be reduced to zero in the region very close to the wall. This was accomplished by multiplying the scaling coefficients of the artificial dissipation terms by the factor, $\left[M I N\left[\left(j / j_{\text {edge }}\right)^{z}, 1\right]\right.$, where $j$ is the index in the $\eta$ direction and $j_{\text {edge }}$ is an estimate of the index of the edge of the boundary layer. $z$ is a positive real number between 2 and 5 . Admittedly the above method is ad hoc and needs to be substituted with a better scheme.

\section{Results and Discussion}

The experimental configurations chosen for comparisons are two for which there is extensive data available. These are: first stage stator from the large low-speed experiments of Dring et al. [19] and stator vane from the experiments of Hylton et al. [3], designated as the $\mathrm{C} 3 \mathrm{X}$ airfoil.

Code validation was performed prior to the above calculations using theoretical correlations available on flat plate [20].

The algebraic and the two-equation models required approximately $40 \times 10^{-6}$ and $85 \times 10^{-6}$ seconds per iteration per grid point respectively. The memory requirements for all the cases were below 2.0 megawords. Computations were performed on the NASA Lewis Cray-XMP. 
The heat transfer parameters were plotted as presented in the respective references. They are defined as follows:

$$
\begin{gathered}
H=\frac{q^{\prime \prime}{ }_{w}}{T_{w}-T_{0(\text { inlet })}} \\
S t=\frac{H}{\rho_{\text {ref }} C_{p} V_{\text {ref }}}
\end{gathered}
$$

where $H$ is the heat transfer coefficient and $q^{\prime \prime}{ }_{w}, T_{w}$ and $T_{0 \text { (inlet) }}$ are the wall heat flux the wall temperature and inlet total temperature respectively. St, $\rho_{\text {ref }}, C_{p}$ and $V_{\text {ref }}$ are the Stanton number, the reference density, heat capacity and reference velocity. The wall heat flux is computed using

$$
q_{w}^{\prime \prime}=-\left.\kappa \frac{\partial T}{\partial n}\right|_{\text {wall }}
$$

\section{Large Low-Speed Cascade}

In this section, the results obtained for the first-stage stator of the large lowspeed cascade of Dring et al. [19] will be discussed. The Reynolds numbers are representative of the conditions in turbomachinery. However, the inlet Mach numbers are consistently below 0.1 in all the experimental runs in [19]. The very low Mach number and the constant wall heat flux boundary condition makes this test require an inordinate number of iterations to converge. The final approach to convergence was extremely slow. It should be noted that the flow pressure converges long before the heat transfer quantities. The number of iterations required for convergence was about 30,000 to 40,000 when the Baldwin-Lomax model was used. The runs using the two-equation model were started from a converged solution using the algebraic model and required an additional 12,000 iterations.

The stator geometry and a typical grid which was employed for the discretization of the equations are presented in figure 1 . The Reynolds number based on the axial 
chord and exit velocity was approximately 250,000 corresponding to the design condition of the cascade. The inlet total temperature and wall heat flux were prescribed as per the experimental conditions. The spacing of the first point away from the wall was chosen such that the value of $y^{+}$was less than unity. Two levels of turbulence were considered, namely, $0.5 \%$ and a grid-generated $9.8 \%$ turbulence level. Figure 2 shows the comparison between the calculated surface pressure variations and the experimental measurements for the above Reynolds number.

Figures 3 and 4 show the experimental and the predicted heat transfer for the two runs of large low-speed cascade considered in this work. Laminar flow calculations are also included for comparison. The experimental results for the low turbulence level of $0.5 \%$ reveal that the flow on the pressure surface is mainly laminar and that only near the trailing edge does it appear to turn transitional. The flow on the suction side, however, does become fully turbulent. The transition to turbulent flow on the suction surface for this case, appears to have been influenced by the change from a favorable to an adverse pressure gradient. In the case of the grid-generated turbulence of $9.8 \%$, the pressure surface flow appears to be in a prolonged transition process. The onset of transition on the suction surface is further upstream as compared to the case of low free stream turbulence. As such it can be concluded that the transition is induced by the free stream turbulence.

Figure 3 shows the results obtained for the low free stream turbulence. For the Baldwin-Lomax model, two grid arrangements were considered, namely, a coarse $150 \times 30$ grid and a fine $200 \times 50$ grid. The Stanton number is based on the exit velocity and density. The fit to the data is satisfactory for both grids. The position of transition from laminar to turbulent flow on the suction side was specified for this calculation using the experimental data. The result presented using the $q-\omega$ model was obtained on the fine grid. The position of transition on the suction surface was not specified and was predicted. The heat transfer agrees well with the experimental data. The pressure surface was predicted to be laminar and heat transfer agrees with 
experiments.

Figure 4 shows the results obtained for the high free stream turbulence. The computation using the Baldwin-Lomax model was performed on the fine grid where the locations of the onset of transition on the suction surface was specified as deduced from the experimental results. The pressure surface boundary layer was assumed to be fully turbulent. Therefore, the onset of transition was specified to be somewhat downstream of the stagnation point. For the two-equation model, an even finer $250 \times$ 50 grid was used. This was done because the flow remained laminar on the pressure surface on a coarser grid. Further refinement of the grid was not performed because of the large computational work required. As can be seen from figure $t$, the suction side transition to turbulent flow as predicted by the Baldwin-Lomax model is abrupt and does not conform to the experimental results. This is because Baldwin-Lomax model does not account for the process of transition. The heat transfer in the turbulent regime is well predicted. The suction surface heat transfer is successfully predicted using the two-equation model. The pressure surface heat transfer is underpredicted for both models and may be due to the coarse grid in the streamwise direction. The stagnation heat transfer for this case is augmented by the high level of free stream turbulence. This cannot be predicted by the present algebraic model. There are algebraic models however, that can account for such effects [10]. The two-equation model correctly predicted the heat transfer near stagnation. This may be fortuitous since it is known that eddy viscosity type models are generally not suitable for the stagnation region. The main reason for the weakness of the two-equation turbulence models in predicting the stagnation flow heat transfer is the use of the Boussinesq hypothesis. This hypothesis works well for shear-dominated flows, but in the case of stagnation flows where the production of turbulence is dominated by the normal stresses, this approximation is inaccurate. 


\begin{tabular}{ccccccc}
\hline \hline Run & $\mathrm{PT}(\mathrm{pa})$ & $\mathrm{TT}\left({ }^{\circ} \mathrm{K}\right)$ & $\mathrm{Tw}\left({ }^{\circ} \mathrm{K}\right)$ & $\mathrm{M} 2$ & $\mathrm{Re}_{2} \times 10^{-6}$ & $\mathrm{Tu} \%$ \\
\hline 149 & 4743 & 795 & 657 & 0.92 & 1.51 & 6.5 \\
158 & 4707 & 808 & 592 & 0.91 & 1.47 & 8.3 \\
109 & 6208 & 796 & 665 & 0.90 & 1.96 & 6.5 \\
113 & 6248 & 781 & 600 & 0.89 & 2.02 & 8.3 \\
\hline \hline
\end{tabular}

Table 1: C3X Cascade Runs

C3X Vane of Hylton et al.

The next set of cases considered was the data of Hylton et al. [3] which were obtained with a cascade of vanes with the designation of C3X. The airfoil is representative of the highly-loaded low-solidity airfoils currently employed. Four runs were chosen such that the effect of the Reynolds number and the inlet free stream turbulence could be studied. The conditions of these runs and their numerical designations are summarized in Table 1 . In the table, TT and PT are the inlet total temperature and pressure respectively and $\mathrm{T} w$ is the wall temperature. The wall to gas temperature ratio, Reynolds and Mach number numbers are representative of engine operating conditions.

Figure 5 shows the cascade and a typical grid employed for the discretization. Other pertinent physical dimensions can be found in reference [3]. The surface pressure variation comparison for Run 149 is presented in figure 6 . In that figure, the abscissa is the distance from the leading edge, normalized by the axial chord. The pressure side experiences a favorable pressure gradient along the surface, while on the suction side the pressure gradient is favorable to approximately the mid-chord where it becomes adverse.

Runs were made using the algebraic model for cases 149 and 109, with two grids of $150 \times 50$ and $200 \times 60$ point densities. Figures 7 and 8 show results obtained using both of the models. Laminar flow and two-equation model calculations are also 
included for comparison. In common with the results presented in [3], the actual heat transfer coefficient is normalized by a reference heat transfer coefficient $H_{0}$ equal to 1135 watts $/ \mathrm{M}^{2} /{ }^{\circ} \mathrm{K}$. The abscissa is the surface distance measured from the stagnation point and is normalized by the suction or the pressure surface arc lengths. The value of $y^{+}$for these runs was consistently chosen to be of the order of 0.5 . The wall boundary condition was constant temperature. Typically, 4,000 iterations were required to obtain converged heat transfer results for the algebraic model.

When using the algebraic model, the locations of the onsets of transition on the the two surfaces were prescribed to best match those of the experiments. The heat transfer is overpredicted when the algebraic model is used. It should be noted however that in reference [3], the flow on the pressure surface is characterized as transitional and not turbulent. This could explain the disagreement between the experimental and the predicted results on the pressure surface.

The two-equation model was run on the fine grid. It was applied to all four cases. The computations typically required an additional 8,000 iterations to converge. Comparisons with the experimental data are shown in figures 9 and 10. The following conclusions were drawn from the results of the two-equation model calculations: 1) The onset of transition from laminar to turbulent flow is correctly predicted for all four cases, however, the heat transfer is underpredicted prior to transition and overpredicted at the end of the transition process; 2) the enhancement of the heat transfer as a result of an increase in the free stream turbulence and wall to gas temperature ratio has been successfully simulated; 3) there is very good agreement for the pressure side for all the four cases; 4) there is an overestimation of the stagnation point heat transfer, the reasons for which have already been explored; 5) the transition from laminar to turbulent flow is not smooth. 


\section{Concluding Remarks}

Predictions were made of the heat transfer rates on turbine vanes using a NavierStokes solver. This is a departure from the common practice of using boundary layer methods to perform this task. The latter requires information on the free stream velocity or pressure which are obtained from experiments or a separate computer program that would solve the Euler equations to extract this information. Other information such as the distribution of the free stream turbulence and the length scale are separately derived by solving the reduced forms of the appropriate equations. The present method does away with that and has the added advantage that it can solve separated flows as well as attached flows. The Baldwin-Lomax algebraic model and Coakely's two-equation model were used in an attempt to account for the turbulence effects. Algebraic models are easy to implement computationally, and do not have the added computational expense of solving the extra model equations and the accompanying convergence difficulties. They are therefore good candidates for a first attempt to estimate the heat transfer coefficients in the turbomachinery environment. The influence of the free stream turbulence on transition from laminar to turbulent flow and its enhancement of heat transfer on the turbomachinery blades is well-known. It was shown that this influence can be accounted for, albeit at a higher cost in computer time, by the use of the two-equation model. There is room for improvements however, as the predictive capability of the model is not equally satisfactory for the cases considered. A more comprehensive study is required to determine the causes of the deficiencies.

Again, it should be stressed that more work is needed to improve the turbulence models, but it is felt that the general approach pursued in this work shows promise for future attempts at obtaining engineering estimates of the heat transfer characteristics of turbomachinery blades. 


\section{Acknowledgement}

The work presented herein, was supported by NASA Lewis Research Center through grant NAG 3-548. 


\section{References}

[1] Patankar, S. V. and Spalding D. B., Heat and Mass Transfer in Boundary Layers, 2nd ed., Intertext, London, 1970.

[2] Crawford, M. E. and Kays, W. M., "STAN5- A Program for Numerical Computation of Two-Dimensional Internal and External Boundary Layer Flows," NASA CR-2742, 1976.

[3] Hylton, L. D., Turner, E. R., Mihelc, M. S., Nealy, D. A. and York, R. E., "Analysis and Experimental Evaluation of the Heat Transfer Distribution over the Surfaces of Turbine Vanes," NASA CR-168015, May 1983.

[4] Wang, J. H., Jen, H. F., and Hartel, E. O., "Airfoil Heat Transfer Calculation Using a Low Reynolds Number Version of a Two-Equation Turbulence Model," Journal of Engineering for Gas Turbine and Power, Vol. 107, Jan. 1985., pp. 60-67.

[5] Rodi, W. and Schenerer, G., "Calculation of Heat Transfer to Convection-Cooled Gas Turbine Blades," Journal of Engineering for Gas Turbines and Power, Vol. 107, July 1985, pp. 620-627.

[6] Schmidt, R. C. and Patankar, S. V., "Two-Equation Low Reynolds Number Turbulence Modeling of Transitional Boundary Layer Flows Characteristic of Gas Turbine Blades," NASA CR-4145, May 1988.

[7] Zerkle, R. D. and Lounsbury, R. J., "The Influence of Freestream Turbulence and Pressure Gradient on Heat Transfer to Gas Turbine Airfoils," AIAA paper 87-1917, 1987.

[8] Carter, J. E. Davis, L. D., Ni R. H., "Cascade Viscous Flow Analysis Using the Navier-Stokes Equations," AIAA paper 86-0033, 1986. 
[9] Marconi, F. and Wilson, G., "The Computation of the Boundary Region Using the Thin-Layer Navier-Stokes Equations," AIAA paper 88-0231, 1988.

[10] Boyle, R. J., "Navier-Stokes Analysis of Turbine Blade External Heat Transfer," ASME Paper 90-GT-42, 35th ASME International Gas Turbine and Aeroengine Conference and Exposition, Brussels, 1990.

[11] Baldwin, B. S. and Lomax, H., "Thin-Layer Approximation and Algebraic Model for Separated Turbulent Flows," AIAA paper 78-0257, 1978.

[12] Coakley, T. J., "Turbulence Modeling Methods for the Compressible Navier-Stokes Equations," AIAA paper 83-1693, 1983.

[13] Chima, R. V., "Development of an Explicit Multigrid Algorithm for Quasi-Three-Dimensional Viscous Flows in Turbomachinery," AIAA paper 86-0032, 1986. (NASA TM-87128, 1986)

[14] Sorenson, R. L., "A Computer Program to Generate Two-Dimensional Grids About Airfoils and Other Shapes by the Use of Poisson's Equation," NASA TM-81198, MAY 1980.

[15] Cebeci, T. and Smith, A.M.O., Analysis of Turbulent Boundary Layers, Academic Press, 1974.

[16] Hah, C., "A Numerical Modeling of Endwall and Tip-Clearance Flow of an Isolated Compressor Rotor," AIAA Journal, Vol. 108, Jan. 1986, pp. 15-21.

[17] Jameson, A., Schmidt, W. and Turkel, E., "Numerical Solutions of the Euler Equations by Finite Volume Methods Using Runge-Kutta TimeStepping Schemes," AIAA paper 81-1259, 1981. 
[18] Tannehill, John C., Anderson, Dale A. and Pletcher, Richard H., Computational Fluid Mechanics and Heat Transfer, Hemisphere Publishing Corporation, New York, 1984, pp. 489-496.

[19] Dring, R. P., Blair, M. F., Joslyn, H. D., Power, G. D., and Verdon, J. M., "The Effect of Inlet Turbulence and Rotor-Stator Interactions on the Aerodynamics and Heat Transfer of a Large-Scale Rotating Turbine Model," NASA CR-4079, 1986.

[20] Ameri, A. A., Navier Stokes Analysis of Turbomachinery Blade External Heat Transfer, Doctoral Dissertation, Cleveland State University, 1990. 
Figure 1: Large Low speed Cascade and a Typical Grid

Figure 2: Pressure Distribution on the Large Low Speed Vane, $R e=250,000$

Figure 3: Stanton No. on the Large Low Speed Vane, $R e=250,000, T u=0.5 \%$

Figure 4: Stanton No. on the Large Low Speed Vane, $R e=250,000, T u=9.8 \%$

Figure 5: C3X Vane Cascade and a Typical Grid

Figure 6: Pressure Distribution on the C3X Vane, Case 149

Figure 7: Heat Transfer Coefficient C3X Vane, Case 149

Figure 8: Heat Transfer Coefficient, C3X Vane, Case 109

Figure 9: Heat Transfer Coefficient, C3X vane, Cases 149 and 158

Figure 10: Heat Transfer Coefficient, C3X Vane, Cases 109 and 113 

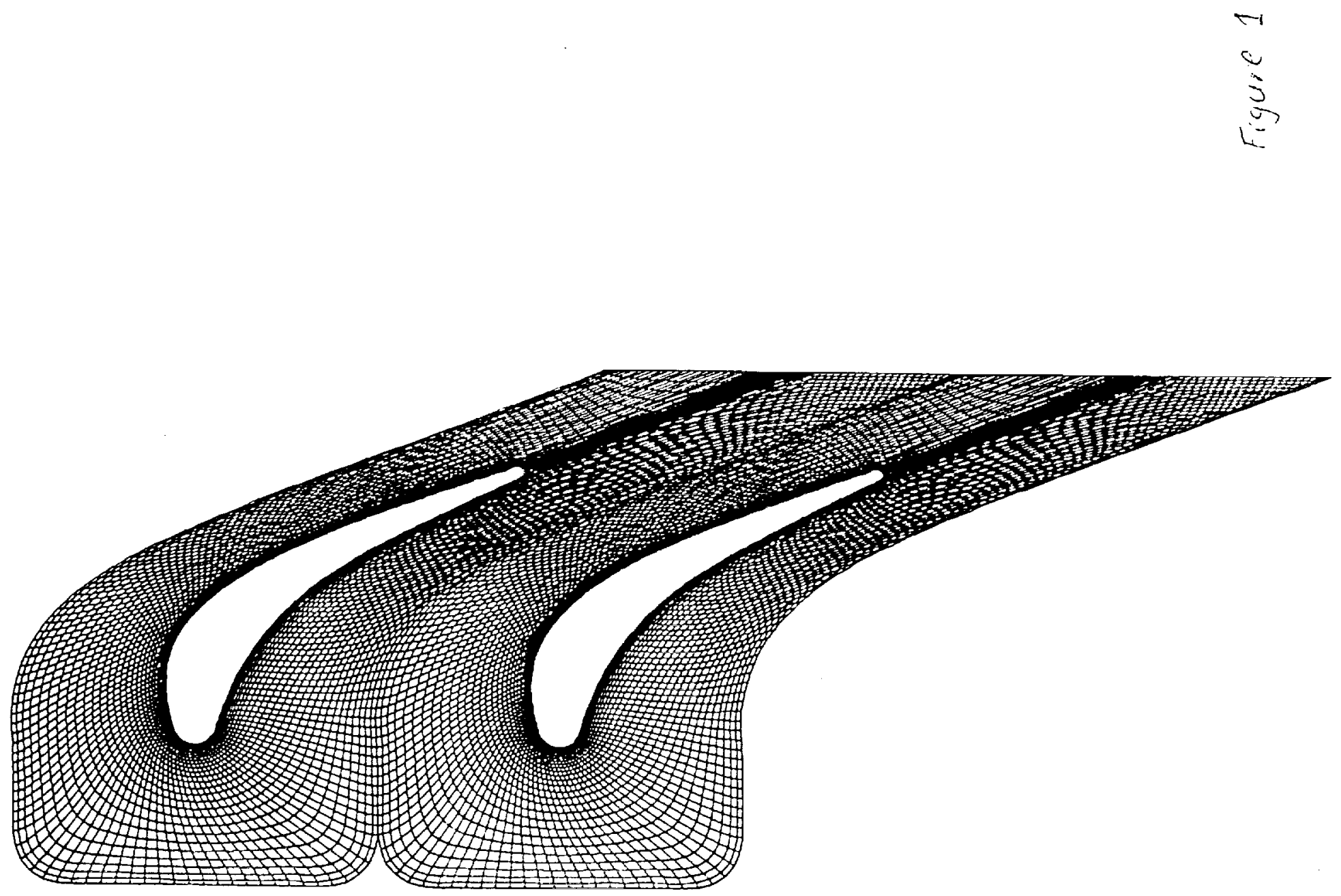


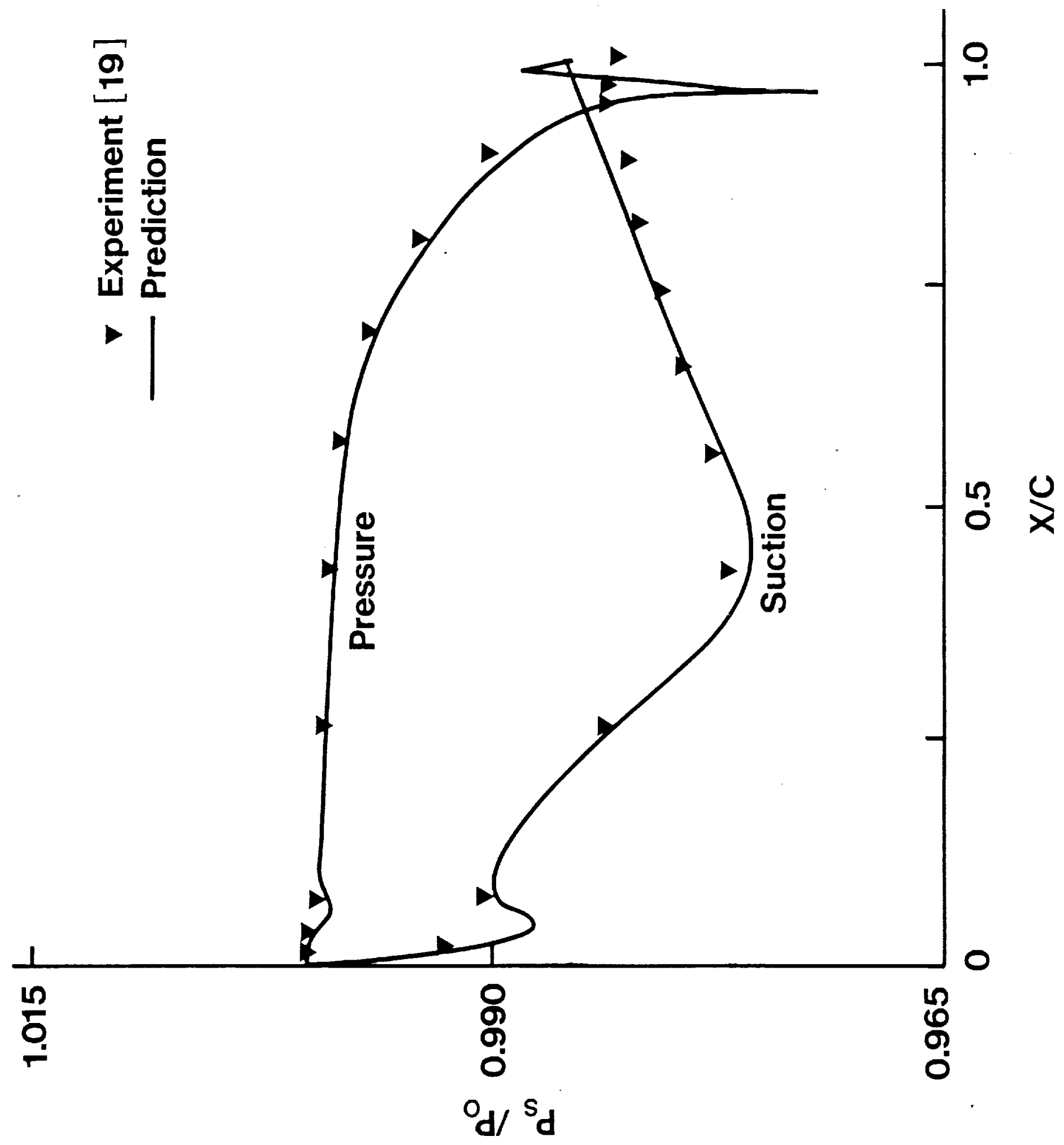




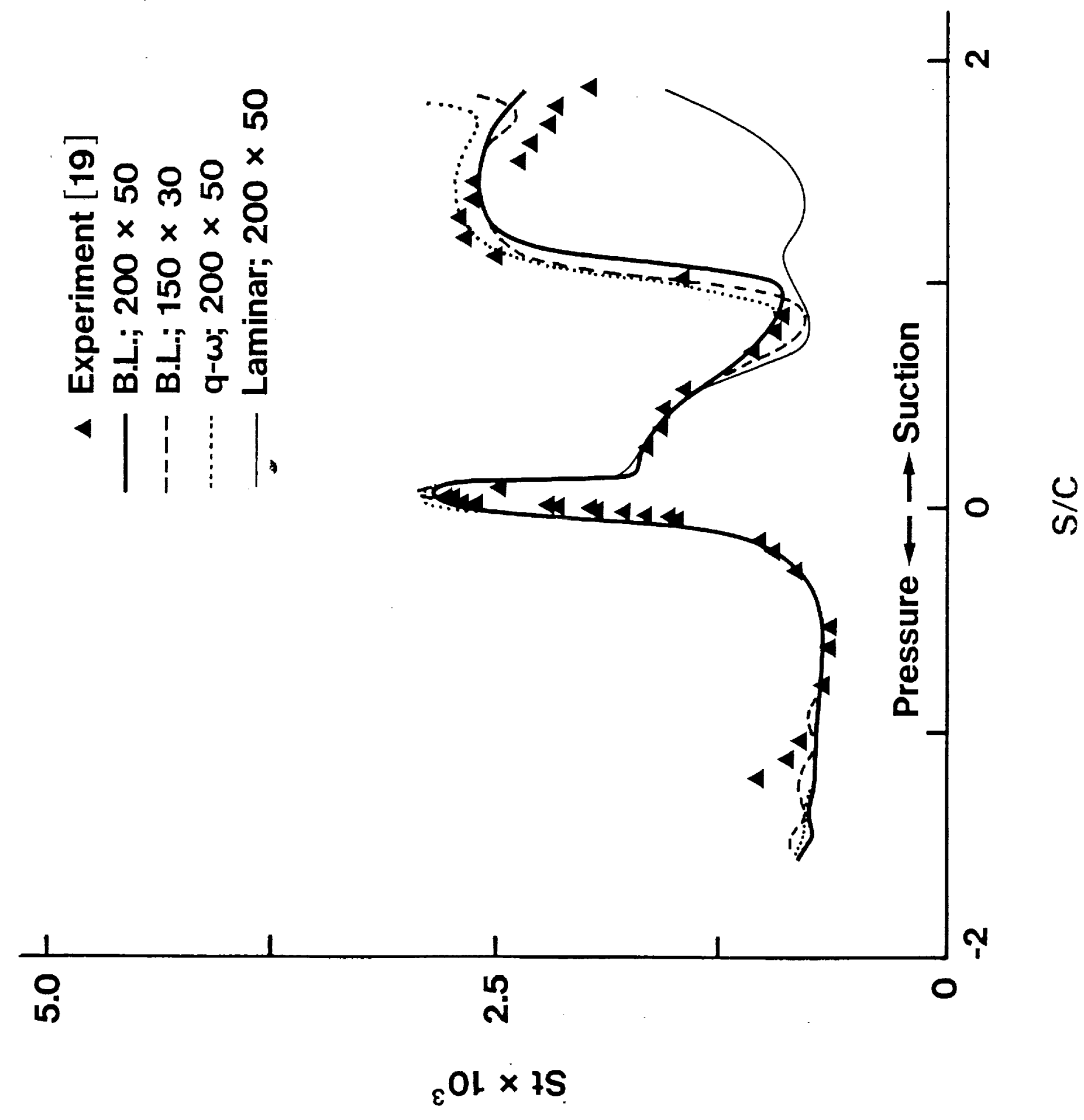




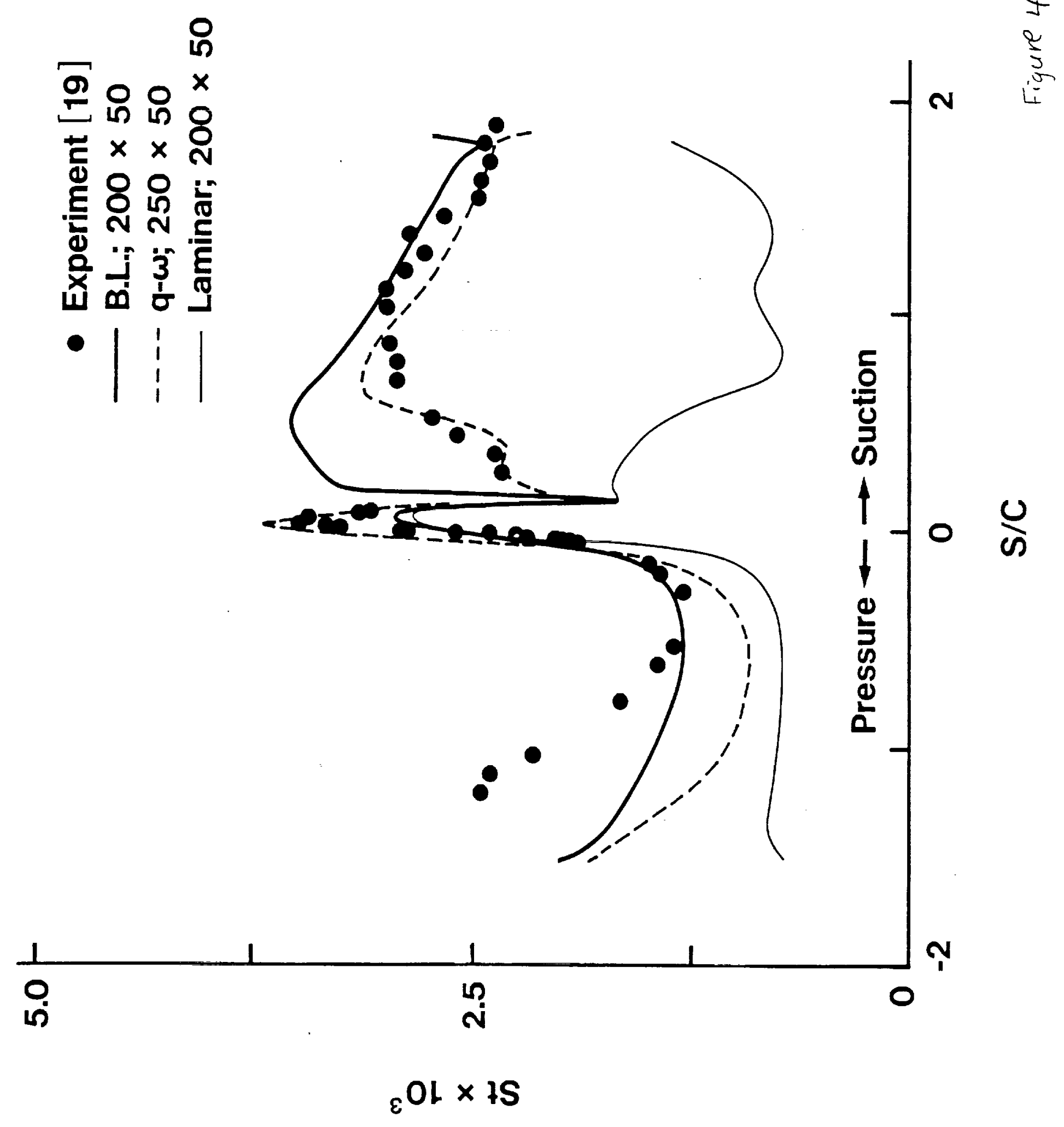




$$
\varnothing
$$




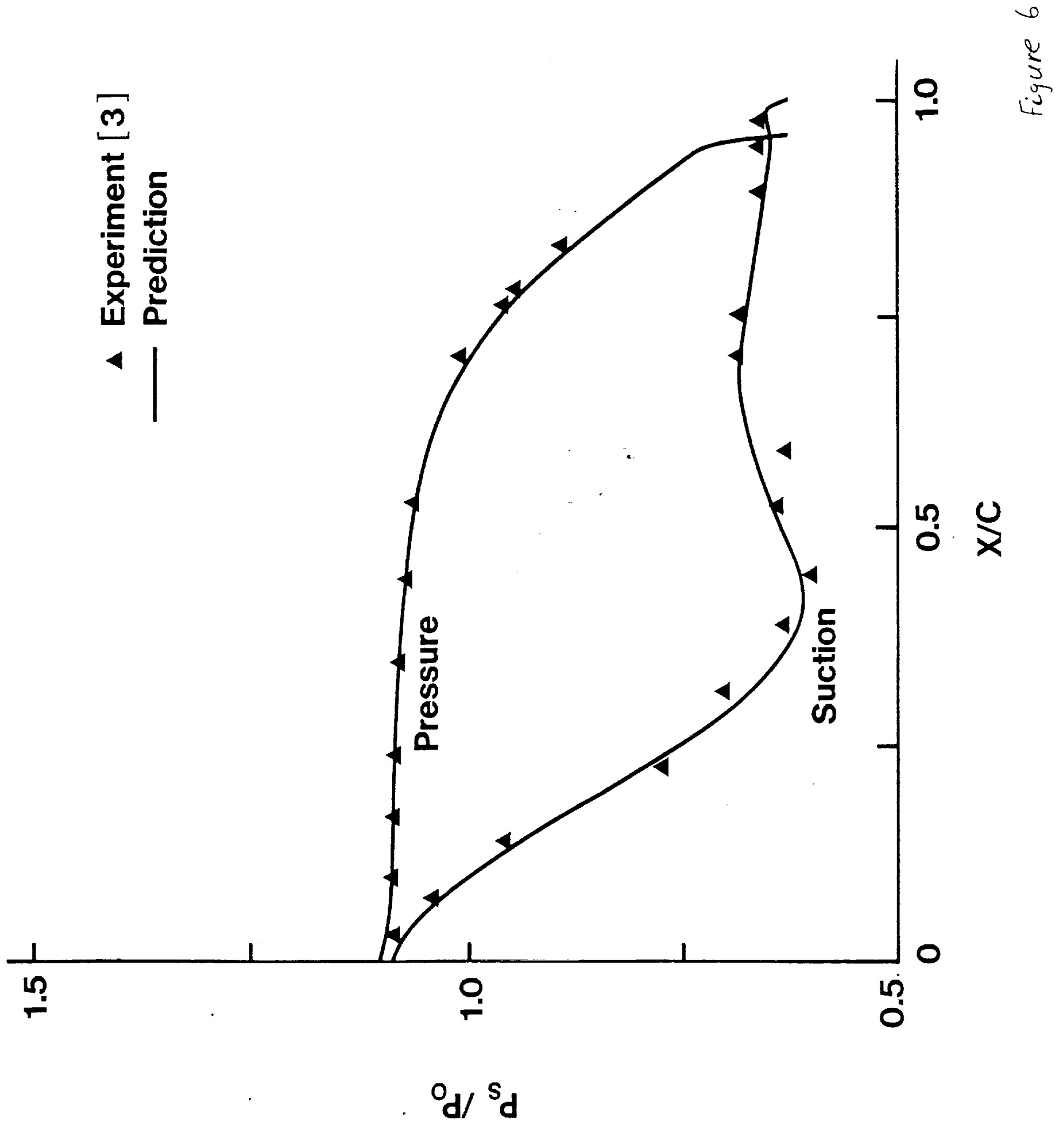




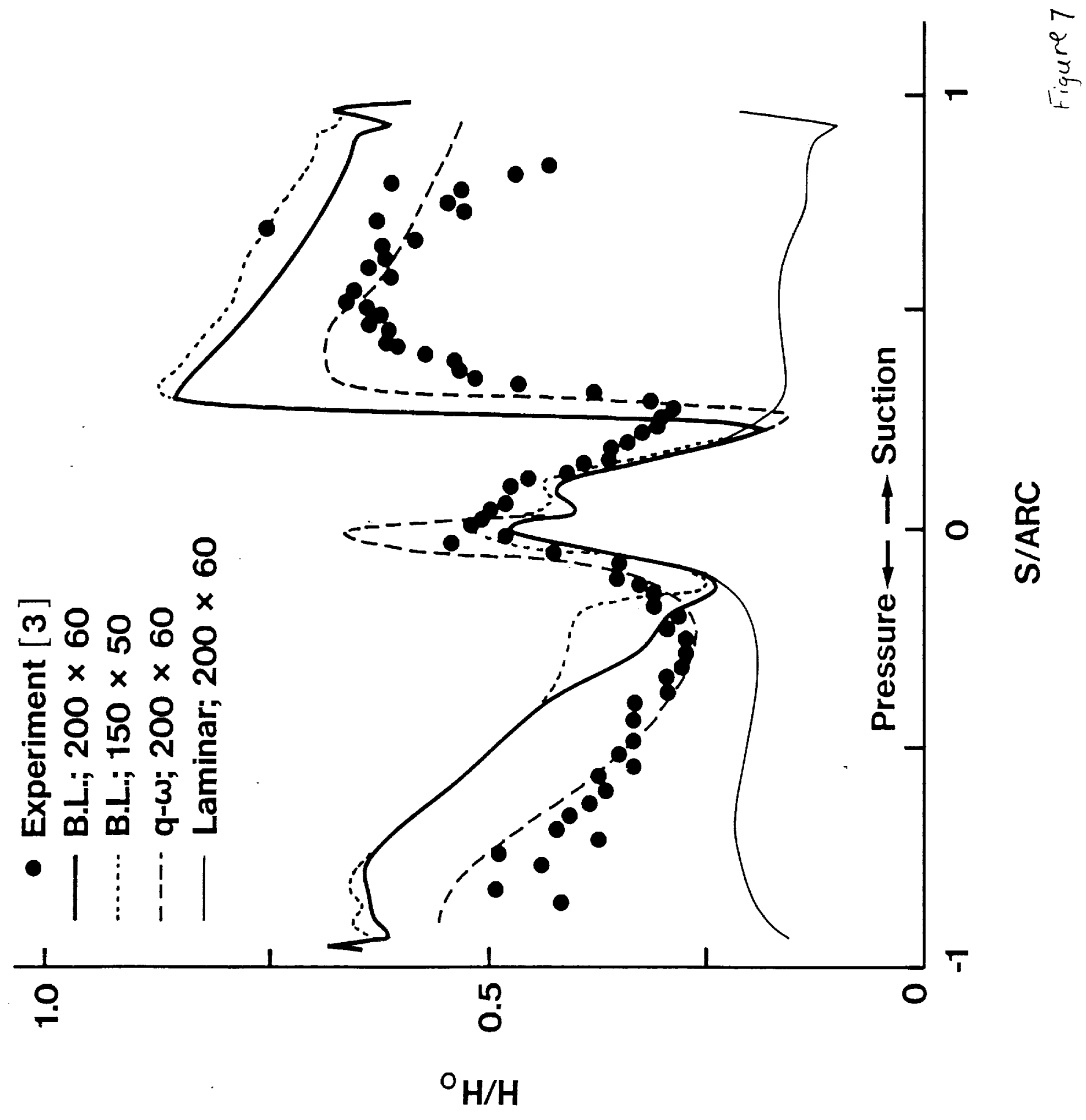




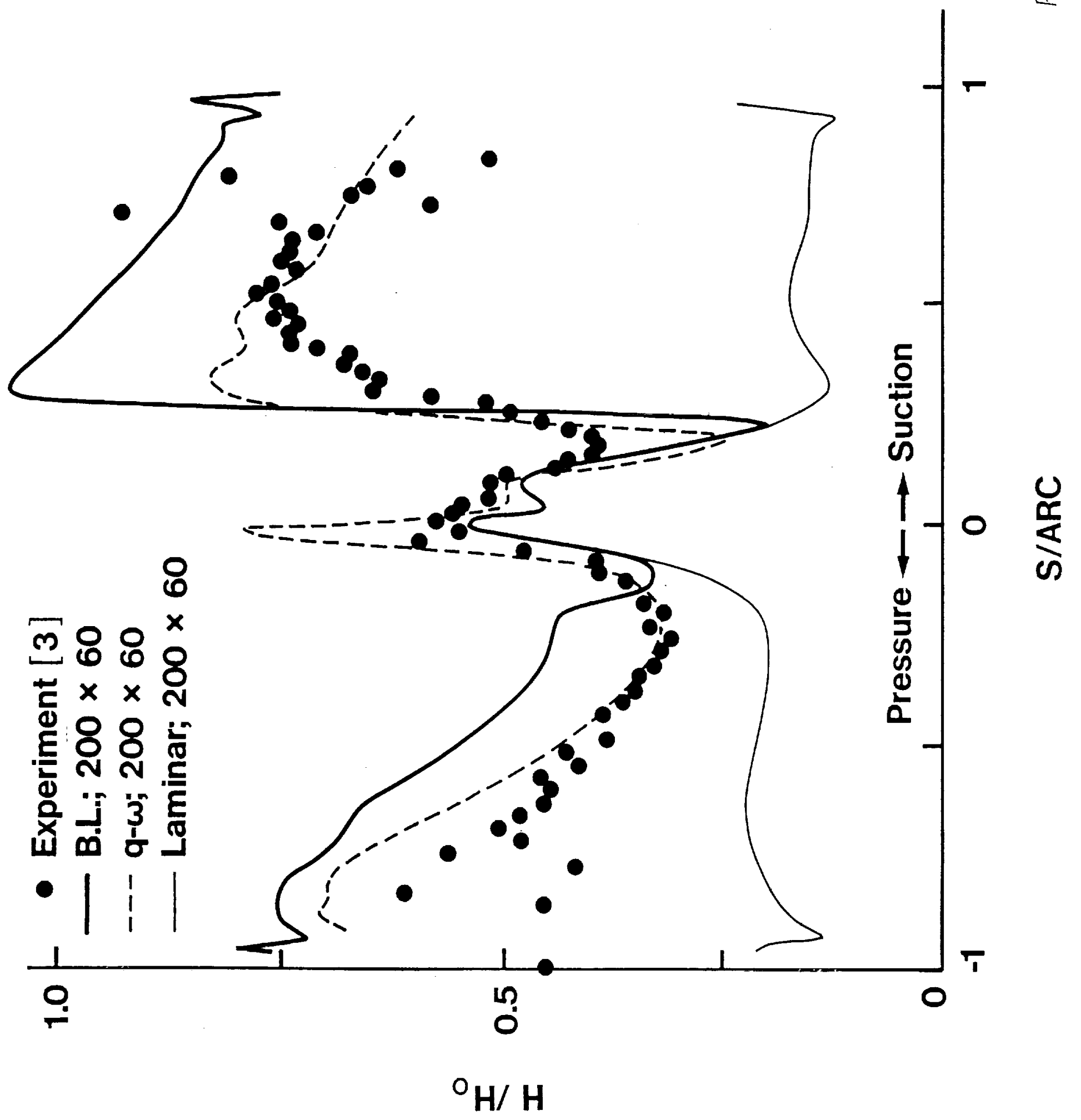




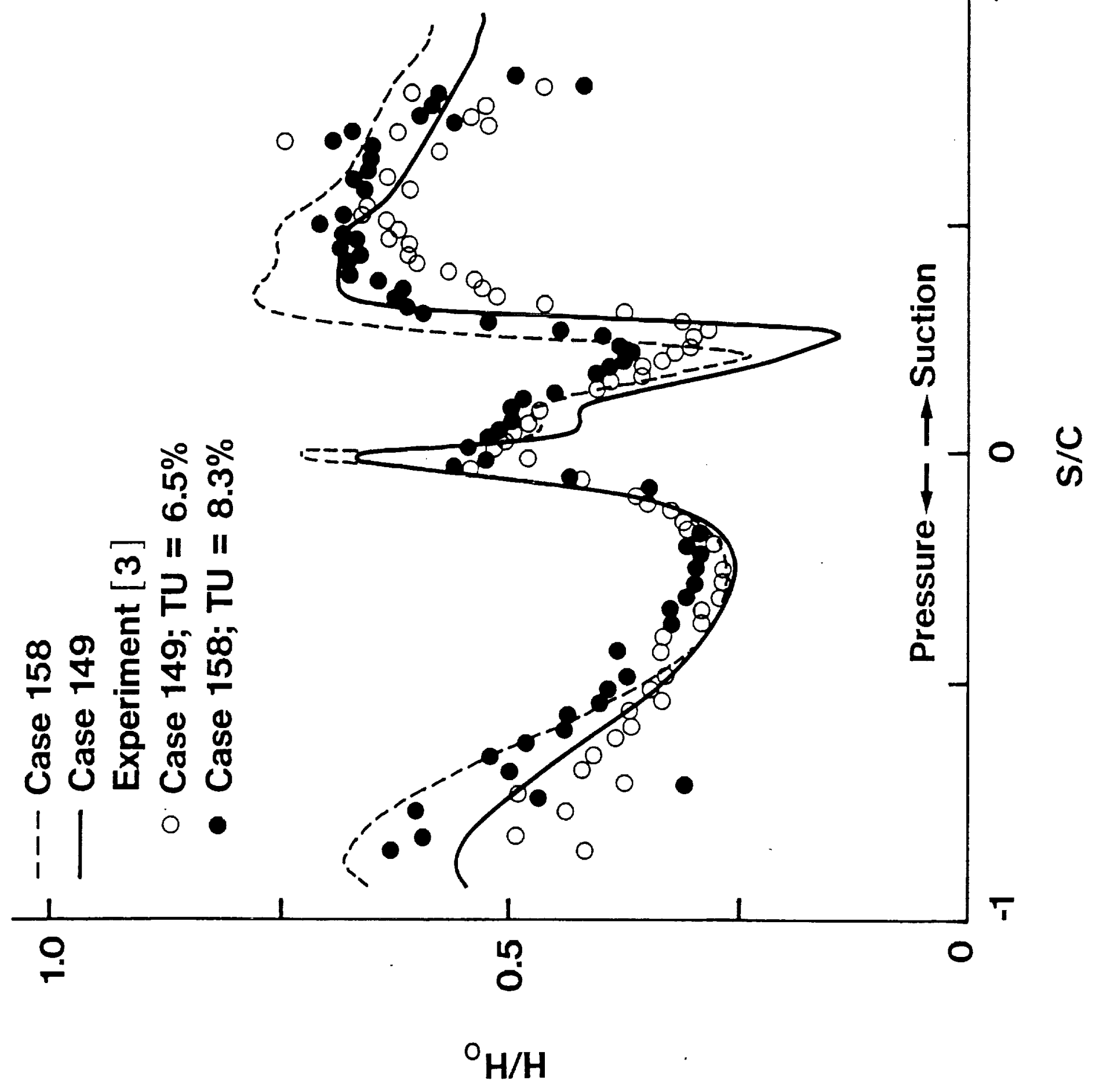




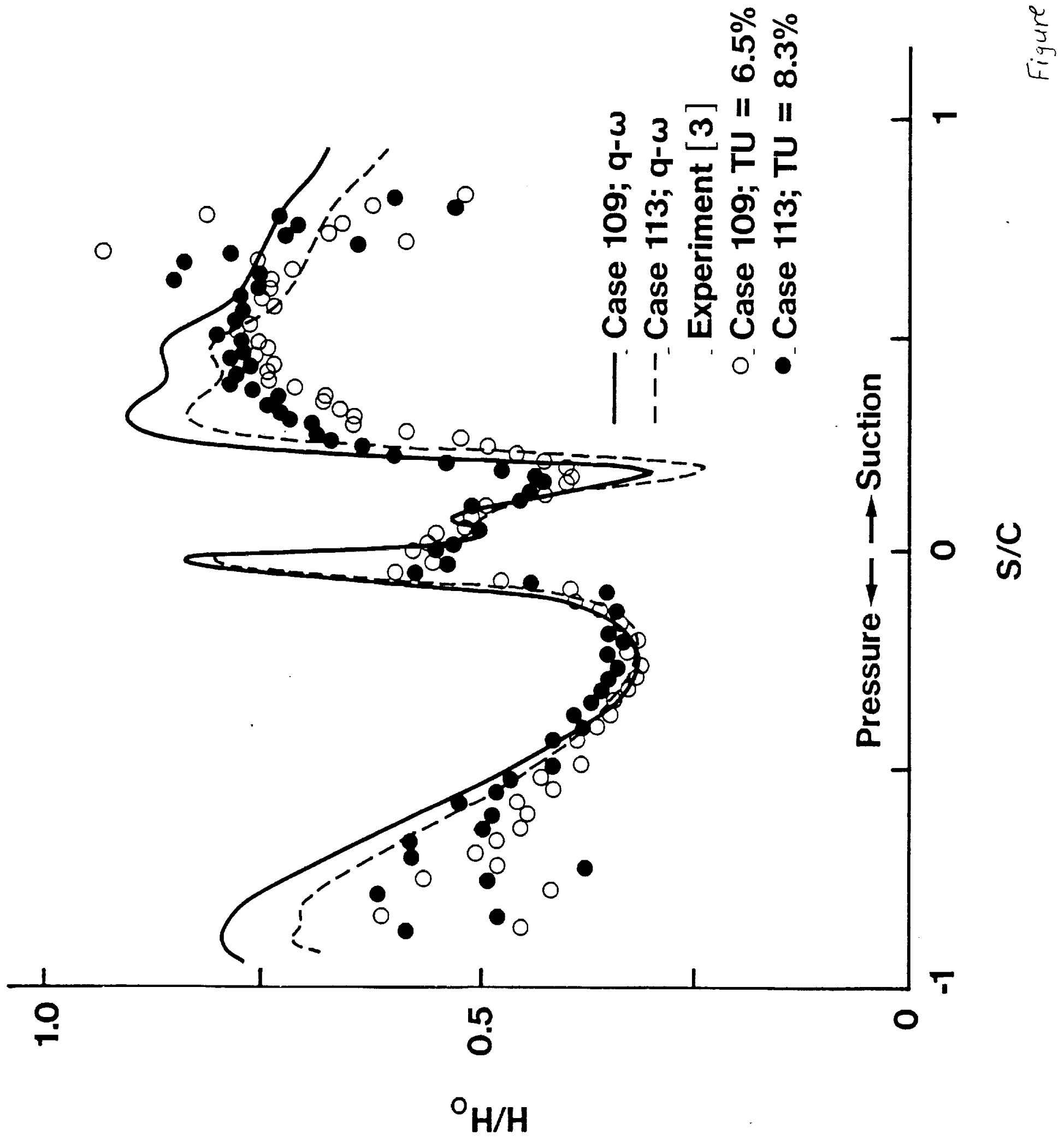

